\title{
Apelin-13 ameliorates metabolic and cardiovascular disorders in a rat model of type 2 diabetes with a high-fat diet
}

\author{
MENG LI ${ }^{1}$, HUIJUAN FANG ${ }^{2}$ and JIAN HU ${ }^{1}$ \\ ${ }^{1}$ Department of Cardiology, The First Affiliated Hospital of China Medical University, Shenyang, Liaoning 110001; \\ ${ }^{2}$ Department of Cadre Ward, The Fourth People's Hospital of Shenyang, Shenyang, Liaoning 110031, P.R. China
}

Received September 13, 2017; Accepted February 19, 2018

DOI: $10.3892 / \mathrm{mmr} .2018 .9607$

\begin{abstract}
Apelin has been reported to be associated with multiple physiological processes in the cardiovascular system. The aim of the present study was to investigate the effects of Apelin-13 administration on cardiac function, hyperglycemia, insulin resistance (IR), dyslipidemia, endothelial function, inflammation and glucose metabolism in type 2 diabetic Goto-Kakizaki (GK) rats, and compare the protective effects of Apelin-13 with metformin or atorvastatin. In the present study, type 2 diabetes was induced in male Goto-Kakizaki (GK) rats fed with high-fat diet (HFD). Simultaneously, the rats were treated with metformin $(350 \mathrm{mg} / \mathrm{kg} / \mathrm{d}$, by gavage), atorvastatin $(50 \mathrm{mg} / \mathrm{kg} / \mathrm{d}$, by gavage) or Apelin-13 $(200 \mu \mathrm{g} / \mathrm{kg} / \mathrm{d}$, intraperitoneal injection) once daily for 4 consecutive weeks. Hemodynamic parameters were examined by RM6240BD multi-channel physiological signal monitoring. Fasting plasma glucose (FPG), fasting insulin (FINS), homeostasis model assessment for insulin resistance (HOMA-IR), total cholesterol (TC), triglyceride (TG), high density lipoprotein-cholesterol (HDL-C), low density lipoprotein-cholesterol (LDL-C), endothelin-1 (ET-1), nitric oxide (NO), constitutive nitric oxide synthase (cNOS) activity, tumor necrosis factor- $\alpha$ (TNF- $\alpha$ ), leptin and Apelin-12 levels were measured. Western blotting was performed to determine the levels of Apelin-12, glucose transporter 4 (GLUT4) and phosphorylated (p)-5'adenosine monophosphate-activated protein kinase (AMPK) $\alpha 2$. It was demonstrated that Apelin-13 decreased heart rate, left ventricular end-diastolic pressure, FPG, FINS, HOMA-IR, TC, TG, LDL-C, ET-1, TNF- $\alpha$ and leptin, whereas it increased the rise and fall of maximum rate of left ventricular pressure, HDL-C, NO, cNOS activity and Apelin-12 compared with the GK-HFD group. In addition, GLUT4 and p-AMPK $\alpha 2$ levels in myocardial tissues were elevated by administration of
\end{abstract}

Correspondence to: Dr Jian Hu, Department of Cardiology, The First Affiliated Hospital of China Medical University, 155 North Nanjing Street, Heping, Shenyang, Liaoning 110001, P.R. China E-mail: hujianyd2017@163.com

Key words: Apelin-13, type 2 diabetes, high-fat diet, metabolic syndrome, Goto-Kakizaki rats, AMP-activated protein kinase
Apelin-13. This protective effect of Apelin-13 was comparable to that of metformin or atorvastatin. Overall, the present study demonstrated that administration of Apelin-13 may be a promising therapeutic agent for the treatment of type 2 diabetes and metabolic syndrome.

\section{Introduction}

Diabetes, characterized by hyperglycemia, is one of the most common chronic diseases (1). Statistical analyses have suggested that 415 million people worldwide suffer from diabetes, and the number of patients will increase to 642 million by $2040(2,3)$. A total of $>90 \%$ of all diabetics are diagnosed with type 2 diabetes (4). Type 2 diabetes is characterized by inadequate insulin secretion from dysfunctional $\beta$ cells and insulin resistance (IR) (4,5). Previous evidence has revealed that diabetes is a predominant risk factor for cardiovascular disease (CVD) (6). Type 2 diabetes is one of the most prevalent diseases in developing and developed countries and is more susceptible to the occurrence of CVD than type 1 diabetes $(7,8)$. Metabolic syndrome, defined as the aggregation of three or more metabolic disorders including obesity, dyslipidemia, hyperglycemia and hypertension, may also increase the risk of type 2 diabetes and CVD $(9,10)$. Currently, the discovery of novel therapeutic agents is still of primary concern for the treatment of type 2 diabetes.

Apelin, an endogenous ligand for angiotensin II protein $\mathrm{J}$ (APJ), was discovered in bovine stomach tissue by Tatemoto et al in 1998 (11). Apelin, a 77-amino acid prepropeptide, can be cleaved into active formsincluding Apelin-12, $-13,-17$ and -36 (12). Apelin is expressed in human plasma, kidney, heart, liver, brain, adipose tissue, gastrointestinal tract and endothelium (13). Apelin/APJ is associated with multiple physiological processes in the cardiovascular system, including enhancement of cardiac contractility, relaxation of blood vessels, and regulation of blood pressure and insulin sensitivity $(14,15)$. Metformin is one of the most widely used drugs in the treatment of type 2 diabetes (16). Atorvastatin has been reported to improve endothelial dysfunction (17). However, whether Apelin-13 has protective effects in high-fat diet (HFD)-induced type 2 diabetes in Goto-Kakizaki (GK) rats remains unclear.

The present study investigated the effects of Apelin-13 administration on cardiac function, hyperglycemia, IR, 
dyslipidemia, endothelial function, inflammation and glucose metabolism in type 2 diabetic GK rats.

\section{Materials and methods}

Animals and grouping. A total of 32 specific-pathogen-free (SPF), male, Goto-Kakizaki (GK) rats (12-weeks-old; 240-280 g) and a total of 8 non-diabetic, male, Wistar rats (12-weeks-old; 240-280 g) were purchased from Shanghai SLAC Laboratory Animal (Shanghai, China). The experiments were performed in accordance with the Guide for the Care and Use of Laboratory Animals and were approved by the Animal Care and Use Committee of China Medical University (Shenyang, China). Ethical clearance was obtained from the Institutional Animal Care and Use Committee (approval no. 2015052R). The animals were maintained under SPF conditions (a 12-h light/dark cycle; temperature, $21 \pm 2^{\circ} \mathrm{C}$; humidity, $60 \pm 10 \%$ ) with access to food and water ad libitum.

Following an adaptive feeding for 1 week, the animals were divided into 5 groups ( $n=8$ rats/group): i) Control, ii) GK-HFD, iii) Metformin, iv) Atorvastatin and v) Apelin-13. Non-diabetic Wistar rats fed with a standard chow and treated with distilled water by gavage were used as the control rats. The GK rats in the GK-HFD, Metformin, Atorvastatin or Apelin-13 group were fed with a high-fat diet (66.5\% standard chow, $10 \%$ lard, 20\% sucrose, $2.5 \%$ cholesterol and $1 \%$ pig bile salt) and given distilled water, metformin $(350 \mathrm{mg} / \mathrm{kg} / \mathrm{d}$, by gavage; Sino-American Shanghai Squibb Pharmaceuticals Ltd., Shanghai, China), atorvastatin $(50 \mathrm{mg} / \mathrm{kg} / \mathrm{d}$, by gavage; Beijing Jialin Pharmaceutical Co., Ltd., Beijing, China) or Apelin-13 (200 $\mu \mathrm{g} / \mathrm{kg} / \mathrm{d}$, intraperitoneal injection; Anaspec Inc., Fremont, CA, USA) once daily for 4 weeks simultaneously.

Hemodynamic parameters. Following 4 weeks of treatment, the rats underwent a $12 \mathrm{~h}$ starvation period. Then, the rats were anesthetized with $3 \%$ pentobarbital sodium $(35 \mathrm{mg} / \mathrm{kg}$; Sinopharm Group Co., Ltd., Shanghai, China) and hemodynamic parameters were monitored using RM6240BD multi-channel physiological signal monitor (Chengdu Instrument Factory, Chengdu, China), including heart rate, left ventricular end diastolic pressure (LVEDP), the maximum rate of left ventricular pressure fall $\left(-\mathrm{dP} / \mathrm{dt}_{\max }\right)$ and maximum rate of left ventricular pressure rise $\left(+\mathrm{dP}^{\left.\mathrm{d} \mathrm{dt}_{\text {max }}\right)}\right.$.

Assessment of biochemical parameters in serum. Fasting venous blood samples were obtained from each rat and serum was obtainedby centrifugation at $1,550 \mathrm{x}$ for $10 \mathrm{~min}$ at $4^{\circ} \mathrm{C}$. Subsequently, serum levels of fasting insulin (FINS; cat. no. F01PZA), endothelin-1 (ET-1; cat. no. D11PZA) and leptin (cat. no. C16PDA) were measured using commercial kits obtained from Beijing North Institute of Biological Technology (Beijing, China). Tumor necrosis factor- $\alpha$ (TNF- $\alpha$; cat. no. XFFM1870) level in serum was examined using a commercial kit from Shanghai Xinfan Biotechnology Co., Ltd. (Shanghai, China). Nitric oxide (NO; cat. no. A012-1) levels and the activity of constitutive nitric oxide synthase (cNOS; cat. no. A014-1-1) were measured using kits obtained from Nanjing Jiancheng Bioengineering Institute (Nanjing, China). All procedures using commercial kits were conducted according to the manufacturer's protocol. Total cholesterol
(TC), triglyceride (TG), high density lipoprotein-cholesterol (HDL-C), low density lipoprotein-cholesterol (LDL-C) levels were determined using a Beckman 700 automatic biochemical analyzer (Beckman Coulter, Inc., Brea, CA, USA).

Determination of fasting plasma glucose (FPG). The animals were deprived of food for $12 \mathrm{~h}$ and blood samples were obtained from the tail vein. The levels of FPG were measured using the ACCU-CHEK Active Glucose Monitoring System (Roche Diagnostics GmbH, Mannheim, Germany) once weekly.

Measurement of homeostasis model assessment for insulin resistance (HOMA-IR). Homeostasis model assessment for insulin resistance (HOMA-IR) was calculated using the following formula: FPGxFINS/22.5.

Apelin-12 expression by ELISA. Apelin-12 expression levels in serum, myocardial tissues and aortic tissues were examined by ELISA according to the manufacturer's protocol (cat. no. EK-057-23; Beijing Shengke Boyuan Biotechnology Co., Ltd., Beijing, China).

Reverse transcription-semi-quantitative polymerase chain reaction $(R T-s q P C R)$ analysis. Myocardial or aortic tissues were lysed with TRIzol ${ }^{\circledR}$ reagent (Invitrogen; Thermo Fisher Scientific, Inc., Waltham, MA, USA) and liquid nitrogen. Total RNAs were isolated from the tissues and RNA concentration was determined by measuring optical density ${ }_{260}$. Subsequently, total RNAs were reverse-transcribed into cDNAs and PCR was performed in a final $25 \mu \mathrm{l}$ volume using a RT-PCR kit (Takara Biotechnology Co., Ltd., Dalian, China). The PCR conditions were: $95^{\circ} \mathrm{C}$ for $5 \mathrm{~min} ; 35$ cycles at $94^{\circ} \mathrm{C}$ for $30 \mathrm{sec}$, $59^{\circ} \mathrm{C}$ for $30 \mathrm{sec}$ and $72^{\circ} \mathrm{C}$ for $40 \mathrm{sec}$, with a final extension at $72^{\circ} \mathrm{C}$ for $5 \mathrm{~min}$. The PCR products were run on $1.2 \%$ agarose gels and stained with $0.5 \mu \mathrm{g} / \mathrm{ml}$ ethidium bromide (Amresco, LLC, Solon, OH, USA). The optical densities of the bands were quantified using a Gel Documentation system (GDS-8000; UVP, LLC, Phoenix, AZ, USA). $\beta$-actin was used as an internal control. The relative gene expression level was normalized to $\beta$-actin levels. All primers were synthesized by Beijing SBS Genetech Co., Ltd (Beijing, China) and the primer sequences were as follows: Forward, 5'-TGCTCTGGCTCTCCTTGA CT-3' and reverse, 5'-ATGGGTCCCTTATGGGAGAG-3' for Apelin-12; forward, 5'-ATCTGGACCCACACCTTC-3' and reverse, 5'-AGCCAGGTCCAGACGCA-3' for $\beta$-actin.

Western blotting. Myocardial tissues were lysed using radioimmunoprecipitation assay lysis buffer, and homogenized using an ultrasonic homogenizer UP200S (Hielscher, Teltow, Germany). Following incubation on ice for $20 \mathrm{~min}$, the tissue homogenates were centrifuged at $4^{\circ} \mathrm{C}$ at $20,000 \mathrm{x}$ for $10 \mathrm{~min}$ and the supernatant was harvested. Protein concentration was determined using the bicinchoninic acid assay method. Proteins were then separated by $10 \%$ SDS-PAGE and transferred onto nitrocellulose membranes (GE Healthcare, Chicago, IL, USA). Following blocking with $5 \%$ non-fat milk at room temperature for $1 \mathrm{~h}$, the membranes were incubated at $4{ }^{\circ} \mathrm{C}$ overnight with primary antibodies against Apelin-12 (1:1,000; cat. no. orb364270; Biorbyt LLC, San Francisco, CA, USA), glucose transporter (GLUT) 4 (1:2,000; cat. no. PA5-19621; Invitrogen; Thermo 

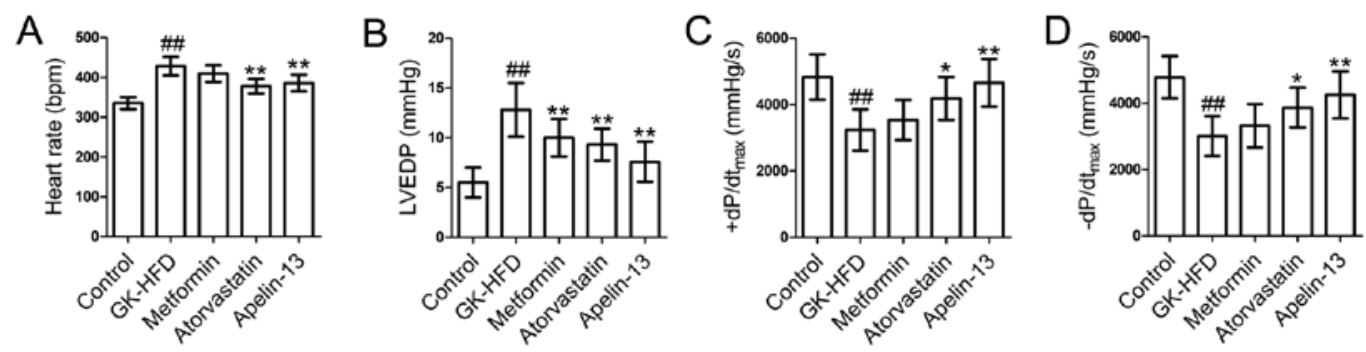

Figure 1. Cardiac function of the rats in each group. Following drug treatment, hemodynamic parameters were monitored to evaluate cardiac function, including (A) heart rate, (B) LVEDP, (C) $+\mathrm{dP} / \mathrm{dt}_{\max }$ and (D) $-\mathrm{dP}^{\mathrm{dt}} \mathrm{t}_{\max }$. Data are expressed as the means \pm standard deviation. ${ }^{\# \#} \mathrm{P}<0.01 \mathrm{vs}$. control group. $\mathrm{P}<0.05$, ${ }^{* *} \mathrm{P}<0.01$ vs. GK-HFD group. GK-HFD, Goto-Kakizaki rats fed with a high-fat diet; LVEDP, left ventricular end-diastolic pressure; $\pm \mathrm{dP}^{\prime} / \mathrm{dt}_{\max }$, rise and fall of maximum rate of left ventricular pressure.

A

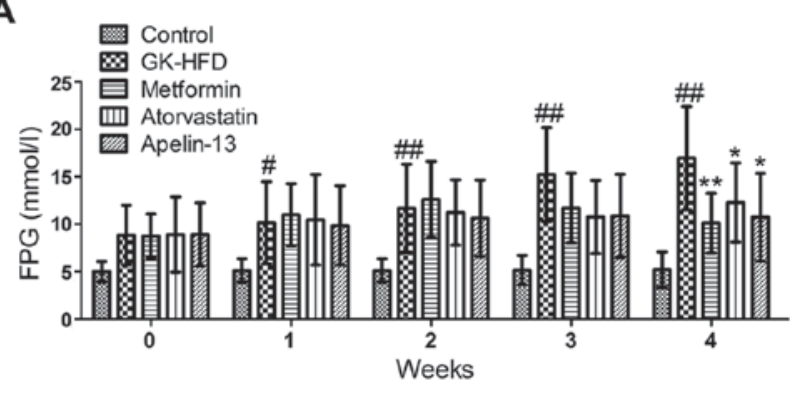

B

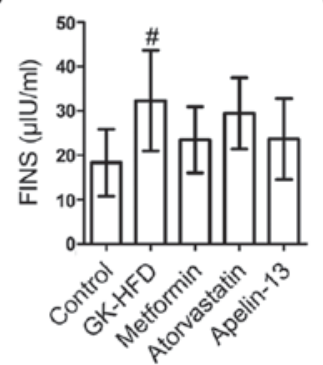

C

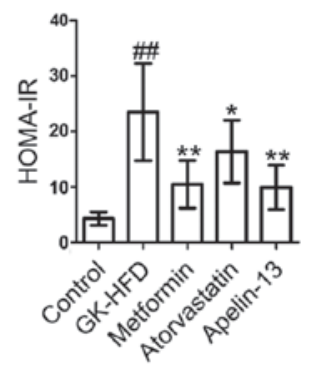

Figure 2. Alterations of FPG, FINS and HOMA-IR. (A) FPG, (B) FINS and (C) HOMA-IR. Data are expressed as the means \pm standard deviation. ${ }^{*} \mathrm{P}<0.05$,

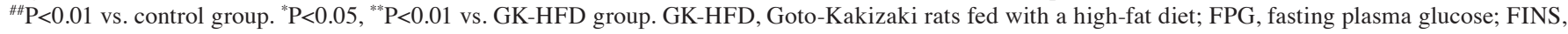
fasting insulin; HOMA-IR, homeostasis model assessment for insulin resistance.

A

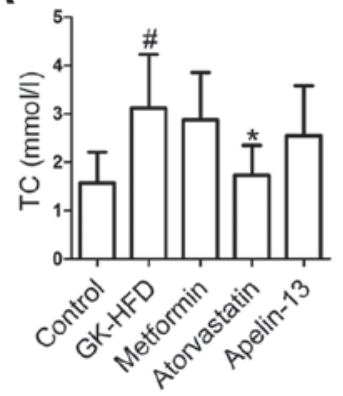

B

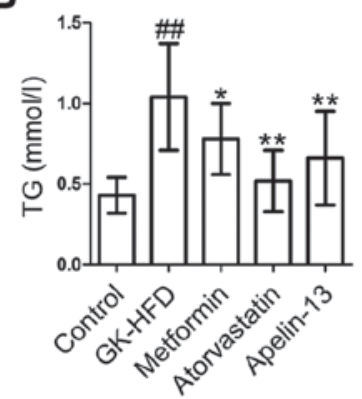

C

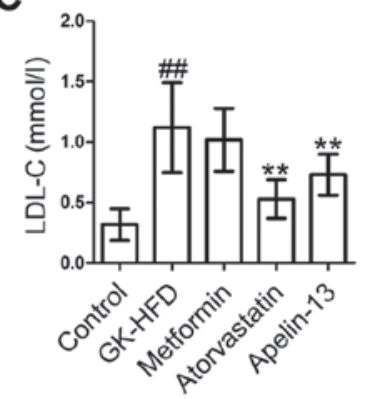

D

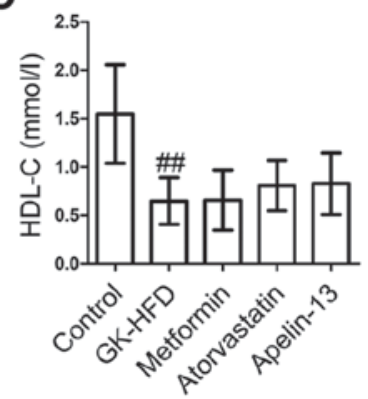

Figure 3. Serum lipid profiles in rats. (A) TC, (B) TG, (C) LDL-C and (D) HDL-C. Data are expressed as the means \pm standard deviation. ${ }^{\#} \mathrm{P}<0.05$, ${ }^{\# \#} \mathrm{P}<0.01$ vs. control group. ${ }^{\mathrm{P}}<0.05,{ }^{* *} \mathrm{P}<0.01$ vs. GK-HFD group. GK-HFD, Goto-Kakizaki rats fed with a high-fat diet; TC, total cholesterol; TG, triglyceride; HDL-C, high density lipoprotein-cholesterol; LDL-C, low density lipoprotein-cholesterol.

Fisher Scientific, Inc.) and phosphorylated (p)-5'adenosine monophosphate-activated protein kinase (AMPK; 1:1,000; cat. no. 18167-1-AP; Wuhan Sanying Biotechnology, Wuhan, China), followed by incubation with horseradish peroxidase-conjugated secondary antibody (1:3,000; cat. no. bs-40296G-HRP; BIOSS, Beijing, China) at room temperature for $1-2 \mathrm{~h}$. Subsequently, protein bands were developed using Super ECL Plus Detection Reagent (cat. no. C05-07004; BIOSS) and quantified using Scion Image software version 1.6.3 (Scion Corporation, Frederick, MD, USA). $\beta$-actin (1:5,000; cat. no. bsm-33036M; BIOSS) served as an internal control.

Statistical analysis. Data were expressed as the means \pm standard deviation. Statistical analyses were performed using GraphPad Prism version 7.0 (GraphPad Software, Inc., La Jolla, CA, USA) with one-way analysis of variance followed by Newman-keuls post-hoc test. $\mathrm{P}<0.05$ was considered to indicate astatistically significant difference.

\section{Results}

Treatment with metformin, atorvastatin and Apelin-13 improves cardiac function. The results demonstrated that heart rate (Fig. 1A) and LVEDP (Fig. 1B) in the GK-HFD group were significantly increased compared with control group, whereas $+\mathrm{dP}^{2} \mathrm{dt}_{\max }$ (Fig. 1C) and $-\mathrm{dP}^{\mathrm{d}} \mathrm{dt}_{\max }$ (Fig. 1D) were decreased compared with control. There was a significant 
A

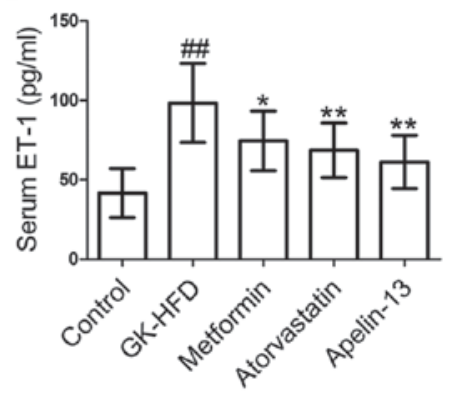

B

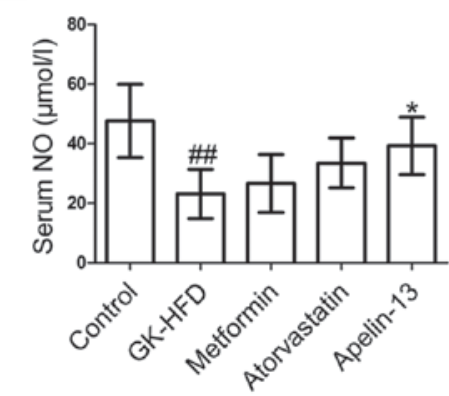

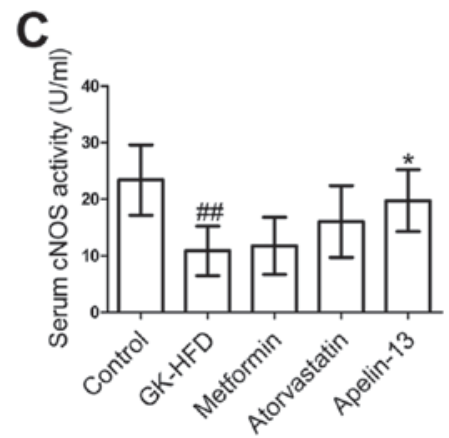

Figure 4. ET-1, NO and cNOS in rats. (A) ET-1 and (B) NO levels in serum. (C) cNOS activity in serum. Data are expressed as the means \pm standard deviation. ${ }^{\# \#} \mathrm{P}<0.01$ vs. control group. ${ }^{*} \mathrm{P}<0.05,{ }^{* *} \mathrm{P}<0.01$ vs. GK-HFD group. GK-HFD, Goto-Kakizaki rats fed with a high-fat diet; ET-1, endothelin-1; NO, nitric oxide; cNOS, constitutive nitric oxide synthase activity.

decrease in heart rate and LVEDP in the Atorvastatin and Apelin-13 groups compared with the GK-HFD group, and an increase in $+\mathrm{dP} / \mathrm{dt}_{\max }$ and $-\mathrm{dP} / \mathrm{dt}_{\max }$. Treatment with metformin significantly decreased LVEDP compared with the GK-HFD group, whereas no statistically significant differences were observed for heart rate and $\pm \mathrm{dP} / \mathrm{dt}_{\max }$.

Treatment with metformin, atorvastatin and Apelin-13 improves insulin resistance. Compared with the control group, the rats in the GK-HFD group had significantly increased levels of FPG (Fig. 2A), FINS (Fig. 2B) and HOMA-IR (Fig. 2C). However, metformin, atorvastatin and Apelin-13 treatment lowered the levels of FPG, FINS and HOMA-IR compared with the GK-HFD group.

Treatment with metformin, atorvastatin and Apelin-13 improves lipid metabolism. The present study then evaluated the effect of metformin, atorvastatin and Apelin-13 on serum levels of TC, TG, LDL-C and HDL-C in rats. The GK-HFD group demonstrated markedly increased levels of TC (Fig. 3A), TG (Fig. 3B) and LDL-C in serum (Fig. 3C) and significantly decreased HDL-C (Fig. 3D) compared with the control group. However, treatment with metformin, atorvastatin and Apelin-13 decreased serum levels of TC, TG and LDL-C and increased HDL-C in GK-HFD rats.

Effect of metformin, atorvastatin and Apelin-13 treatment on ET-1, NO and cNOS. The ET-1 level in serum (Fig. 4A) was significantly increased in the GK-HFD group compared with the control group, however was significantly decreased in the Metformin, Atorvastatin and Apelin-13 groups, compared with GK-HFD group. In addition, the GK-HFD group exhibited a significant decrease in serum NO level (Fig. 4B) and a reduction in cNOS activity (Fig. 4C). However, metformin, atorvastatin and Apelin-13 administration elevated NO level and cNOS activity.

Effect of metformin, atorvastatin and Apelin-13 treatment on serum TNF- $\alpha$ and leptin. Compared with the control rats, GK-HFD rats exhibited a significant increase in TNF- $\alpha$ (Fig. 5A) and leptin (Fig. 5B) in serum. However, the serum levels of TNF- $\alpha$ and leptin in the Metformin, Atorvastatin and Apelin-13 groups were significantly decreased, compared with the GK-HFD group.
A

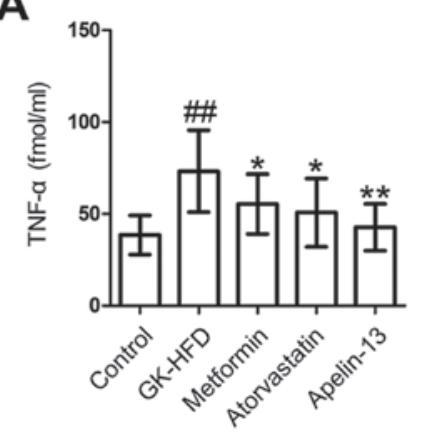

B

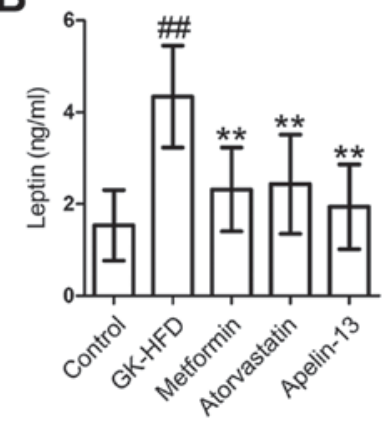

Figure 5. Alterations of TNF- $\alpha$ and leptin serum levels in rats. (A) TNF- $\alpha$ levels in serum. (B) Leptin levels in serum. Data are expressed as the means \pm standard deviation. ${ }^{\# \#} \mathrm{P}<0.01$ vs. control group. ${ }^{*} \mathrm{P}<0.05,{ }^{* *} \mathrm{P}<0.01$ vs. GK-HFD group. GK-HFD, Goto-Kakizaki rats fed with a high-fat diet; TNF- $\alpha$ tumor necrosis factor- $\alpha$.

Effect of metformin, atorvastatin and Apelin-13 treatment on Apelin-12 expression. Following this, the expression levels of Apelin-12 in serum, myocardial tissues and aortic tissues were measured. As presented in Fig. 6, the levels of Apelin-12 in myocardial tissues (Fig. 6A, D and F), aortic tissues (Fig. 6B and E) and serum (Fig. 6C) in the GK-HFD group were significantly decreased compared with the control group. However, treatment with metformin, atorvastatin and Apelin-13 significantly induced the expression of Apelin-12 in serum, myocardial tissues and aortic tissues, compared with the GK-HFD group.

Effect of metformin, atorvastatin and Apelin-13 treatment GLUT4 and $p$-AMPKa2. The levels of GLUT4 and p-AMPK $\alpha 2$ (Fig. 6F) in the myocardial tissues of GK-HFD rats were significantly decreased compared with control group. However, metformin and Apelin-13 injections markedly elevated GLUT4 and p-AMPK $\alpha 2$ levels, compared with the GK-HFD group. Atorvastatin treatment resulted in slight increases in GLUT4 and p-AMPK 22 levels; however, the differences were not statistically significant.

\section{Discussion}

The GK rat may be used as a genetic animal model of type 2 diabetes (18). In the present study, type 2 diabetes 

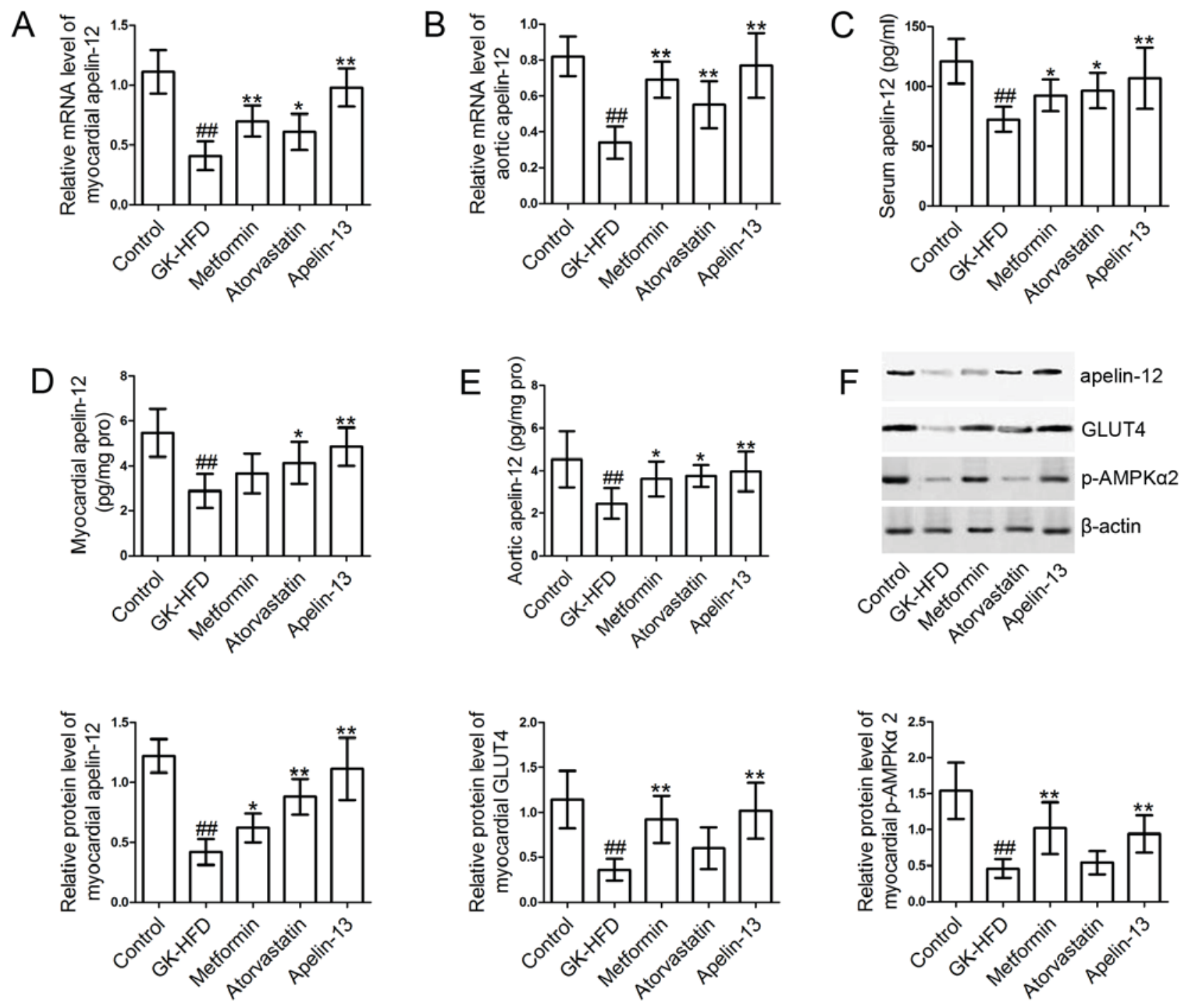

Figure 6. Apelin-12, GLUT4 and p-AMPKa2 expression levels in rats. Apelin-12 mRNA levels in (A) myocardial and (B) aortic tissues were measured via reverse transcription-semi-quantitative polymerase chain reaction. Apelin-12 levels in (C) serum, (D) myocardial and (E) aortic tissues were determined using ELISA. (F) Western blotting analyses of Apelin-12, GLUT4 and p-AMPK $\alpha 2$ in myocardial tissues. $\beta$-actin served as the internal control. Data are expressed as the means \pm standard deviation. ${ }^{\# \#} \mathrm{P}<0.01$ vs. control group. ${ }^{*} \mathrm{P}<0.05,{ }^{* *} \mathrm{P}<0.01$ vs. GK-HFD group. GK-HFD, Goto-Kakizaki rats fed with a high-fat diet; GLUT4, glucose transporter 4; p, phosphorylated; AMPK $\alpha 2,5$ 'adenosine monophosphate-activated protein kinase $\alpha 2$.

was induced in GK rats. The effects of metformin, atorvastatin and Apelin-13 on cardiac function, hyperglycemia, IR, dyslipidemia, endothelial function, inflammation and glucose metabolism in GK-HFD rats were investigated.

Hemodynamic indices, including HR, LVEDP, $+\mathrm{dP} / \mathrm{dt}_{\max }$ and $-\mathrm{dP} / \mathrm{dt}_{\max }$, are often used to evaluate cardiac function (19). $+\mathrm{dP} / \mathrm{dt}_{\max }$ indicates systolic cardiac function, whereas LVEDP and $-\mathrm{dP} / \mathrm{dt}_{\max }$ indicate diastolic cardiac function (20). The results demonstrated that Apelin-13 decreased heart rate and LVEDP, and increased $+\mathrm{dP}^{2} \mathrm{dt}_{\max }$ and $-\mathrm{dP} / \mathrm{dt}_{\max }$, indicating the improvement of LV systolic and diastolic function in GK rats fed with HFD.

IR and high FPG are important risk factors for type 2 diabetes $(21,22)$. HOMA-IR, calculated from FPG and FINS, is commonly used as a primary index for IR evaluation in the prevention of diabetes and screening of high-risk groups (23). The HOMA-IR was used to determine IR on the basis of fasted insulin and glucose levels. Elevated HOMA-IR has a positive impact on the development of type 2 diabetes in patients with impaired insulin secretion (24). In the present study, it was demonstrated that Apelin-13 significantly reduced the elevated FPG, FINS and HOMA-IR. The results suggested that Apelin-13 improved IR in the rat model of type 2 diabetes.

Dyslipidemia, characterized by increases in TC, TG and LDL-C, and decreases in HDL-C, is a common disorder in type 2 diabetics $(25,26)$. TC and TG, two predominant types of lipids in plasma, are predictors of the balance of lipid metabolism (27). Elevated plasma HDL-C results in a cardioprotective effect, whereas higher LDL-C levels are considered an atherogenic factor (28). In the present study, it was demonstrated that Apelin-13 decreased TC, TG and LDL-C, and increased HDL-C concentration in serum, which indicated that Apelin-13 improved dyslipidemia in a rat model of type 2 diabetes.

Endothelial dysfunction has a vital role in the progression of diabetic vasculopathy and hypertension (29). It has been reported that ET-1 is overproduced in animal models of diabetes and patients (30). ET-1 is primarily produced in 
the endothelium, cardiomyocytes, vascular smooth muscle cells, fibroblasts, leukocytes and macrophages (31). ET-1, which is a potent peptide vasoconstrictor with proinflammatory and profibrotic properties, participates in the development of diabetic vasculopathy via regulation of vascular homeostasis $(30,32,33)$. NO is important in various physiological processes. cNOS produces a small amount of NO and has been reported to be associated with $\beta$-cell dysfunction during the development of type 2 diabetes (34). In addition, a previous study revealed that overproduction of ET-1 may contribute to endothelial dysfunction by inhibiting NO secretion (31). The results of the present study demonstrated that Apelin-13 injection resulted in decreased levels of ET-1 and increased NO serum levels and cNOS activity. These results suggested that Apelin-13 alleviated endothelial dysfunction by regulating the imbalance of ET-1 secretion and NO production.

Diabetes is an inflammatory disease in which the levels of pro-inflammatory cytokines, including $\mathrm{TNF}-\alpha$, are elevated in the serum of patients $(35,36)$. A previous study indicated that high levels of TNF- $\alpha$ is a crucial risk factor for diabetes (37). Furthermore, TNF- $\alpha$ is an important indicator of insulin resistance in obesity and may serve as a target for improving obesity-induced insulin resistance in patients with type 2 diabetes (38). Leptin, first discovered by Zhang et al in 1994, is a hormone that is secreted from adipose tissues and circulates in the blood $(39,40)$. Leptin controls body weight and adipose tissue mass via regulation of energy homeostasis (41). Patients with obesity and type 2 diabetes usually have an increased plasma level of leptin and leptin resistance results in a failure to improve hyperglycemia (42). In addition, elevated leptin levels in plasma are correlated with IR, independent of insulin sensitivity and obesity (41). It was demonstrated that Apelin-13 injection reduced the increased levels of TNF- $\alpha$ and leptin induced by diabetes. These results suggested that Apelin-13 may alleviate diabetic disorders via inhibition of TNF- $\alpha$ and leptin secretion.

A previous study suggested that the circulating levels of Apelin are decreased in patients with type 2 diabetes (43). Apelin, associated with glucose uptake and IR, may promote the translocation of GLUT4 from the cytoplasm to the plasma membrane (44). GLUT4 is primarily present in cardiac, adipose and skeletal tissues, and is an insulin-regulated glucose transporter (45). Glucose is transported across the cell membrane via glucose transporters (GLUTs). GLUT4 is reported to be decreased in diabetic patients, which leads to a decrease in the uptake/utilization of glucose; whereas, cardiac contractility and metabolism are improved when GLUT4 is upregulated (46). AMPK, a conserved serine/threonine protein kinase, acts as a target for metabolic syndrome prevention (47). In the present study, it was demonstrated that Apelin-13 elevated Apelin-12 expression in serum, myocardial tissues and aortic tissues and resulted in increases in myocardial GLUT4 and p-AMPK $\alpha 2$ levels. These results indicated that Apelin-13 may enhance glucose metabolism and activate the AMPK signaling pathway.

In conclusion, the results of the present study demonstrated that Apelin-13 exerted beneficial effects on cardiac function, hyperglycemia, IR, dyslipidemia, endothelial function, inflammation and glucose metabolism, via upregulation of Apelin-12 and activation of the AMPK signaling pathway in type 2 diabetes. This protective effect of Apelin was comparable to that of metformin or atorvastatin. These findings indicate that Apelin-13 may be a potential therapeutic agent for the treatment of type 2 diabetes.

\section{Acknowledgements}

Not applicable.

\section{Funding}

No funding was received.

\section{Availability of data and materials}

All data generated or analyzed during this study are included in this published article.

\section{Authors' contributions}

ML and JH conceived the study and designed the experiments. ML and HJF performed the experiments and analyzed the data. ML and HJF provided reagents, materials and analysis tools. ML and JH wrote and revised the manuscript.

\section{Ethics approval and consent to participate}

The experiments were performed in accordance with the Guide for the Care and Use of Laboratory Animals and were approved by the Animal Care and Use Committee of China Medical University (Shenyang, China). Ethical clearance was obtained from the Institutional Animal Care and Use Committee (approval no. 2015052R).

\section{Patient consent for publication}

Not applicable.

\section{Competing interests}

The authors declare that they have no competing interests.

\section{References}

1. Yakaryilmaz FD and Ozturk ZA: Treatment of type 2 diabetes mellitus in the elderly. World J Diabetes 8: 278-285, 2017.

2. Koch A, Grammatikos G, Trautmann S, Schreiber Y, Thomas D, Bruns F, Pfeilschifter J, Badenhoop K and Penna-Martinez M: Vitamin D supplementation enhances c18(dihydro)ceramide levels in type 2 diabetes patients. Int J Mol Sci 18: E1532, 2017.

3. Bilir B, Ekiz Bilir B, Yilmaz I, Soysal Atile N, Yildirim T, Kara SP, Gumustas SA, Orhan AE and Aydin M: Association of apelin, endoglin and endocan with diabetic peripheral neuropathy in type 2 diabetic patients. Eur Rev Med Pharmacol Sci 20: 892-898, 2016.

4. Peiro C, Lorenzo O, Carraro R and Sanchez-Ferrer CF: IL-1 $\beta$ inhibition in cardiovascular complications associated to diabetes mellitus. Front Pharmacol 8: 363, 2017.

5. Dunn JS, Mlynarski WM, Pezzolesi MG, Borowiec M, Powers C, Krolewski AS and Doria A: Examination of PPP1R3B as a candidate gene for the type 2 diabetes and MODY loci on chromosome 8p23. Ann Hum Genet 70: 587-593, 2006. 
6. Tun NN, Arunagirinathan G, Munshi SK and Pappachan JM Diabetes mellitus and stroke: A clinical update. World J Diabetes 8: 235-248, 2017.

7. Shukla SK, Liu W, Sikder K, Addya S, Sarkar A, Wei Y and Rafiq K: HMGCS2 is a key ketogenic enzyme potentially involved in type 1 diabetes with high cardiovascular risk. Sci Rep 7: 4590, 2017.

8. Naqvi S, Naveed S, Ali Z, Ahmad SM, Asadullah Khan R, Raj H, Shariff S, Rupareliya C, Zahra F and Khan S: Correlation between glycated hemoglobin and triglyceride level in type 2 diabetes mellitus. Cureus 9: e1347, 2017.

9. Kupelian V, Hayes FJ, Link CL, Rosen R and McKinlay JB: Inverse association of testosterone and the metabolic syndrome in men is consistent across race and ethnic groups. J Clin Endocrinol Metab 93: 3403-3410, 2008.

10. Saibandith B, Spencer JPE, Rowland IR and Commane DM: Olive polyphenols and the metabolic syndrome. Molecules 22 E1082, 2017.

11. Tatemoto K, Hosoya M, Habata Y, Fujii R, Kakegawa T, Zou MX, Kawamata Y, Fukusumi S, Hinuma S, Kitada C, et al: Isolation and characterization of a novel endogenous peptide ligand for the human APJ receptor. Biochem Biophys Res Commun 251: 471-476, 1998.

12. Cirillo P, Ziviello F, Pellegrino G, Conte S, Cimmino G, Giaquinto A, Pacifico F, Leonardi A, Golino P and Trimarco B: The adipokine apelin-13 induces expression of prothrombotic tissue factor. Thromb Haemost 113: 363-372, 2015.

13. Folino A, Montarolo PG, Samaja M and Rastaldo R: Effects of apelin on the cardiovascular system. Heart Fail Rev 20: 505-518, 2015.

14. Zhou Q, Cao J and Chen L: Apelin/APJ system: A novel therapeutic target for oxidative stress-related inflammatory diseases (Review). Int J Mol Med 37: 1159-1169, 2016.

15. Huang S, Chen L, Lu L and Li L: The apelin-APJ axis: A novel potential therapeutic target for organ fibrosis. Clin Chim Acta 456: 81-88, 2016

16. Wang YW, He SJ, Feng X, Cheng J, Luo YT, Tian L and Huang Q Metformin: A review of its potential indications. Drug Des Devel Ther 11: 2421-2429, 2017

17. Sena CM, Matafome P, Louro T, Nunes E and Seica RM: Effects of atorvastatin and insulin in vascular dysfunction associated with type 2 diabetes. Physiol Res 63: 189-197, 2014.

18. Howarth FC, Qureshi MA, Sobhy ZH, Parekh K, Yammahi SR, Adrian TE and Adeghate E: Structural lesions and changing pattern of expression of genes encoding cardiac muscle proteins are associated with ventricular myocyte dysfunction in type 2 diabetic goto-kakizaki rats fed a high-fat diet. Exp Physiol 96 765-777, 2011.

19. Liu Y, Qi H, Wang Y, Wu M, Cao Y, Huang W, Li L, Ji Z and Sun H: Allicin protects against myocardial apoptosis and fibrosis in streptozotocin-induced diabetic rats. Phytomedicine 19: 693-698, 2012

20. Wichi R, Malfitano C, Rosa K, De Souza SB, Salemi V, Mostarda C, De Angelis K and Irigoyen MC: Noninvasive and invasive evaluation of cardiac dysfunction in experimental diabetes in rodents. Cardiovasc Diabetol 6: 14, 2007.

21. Er LK, Wu S, Chou HH, Hsu LA, Teng MS, Sun YC and Ko YL: Triglyceride glucose-body mass index is a simple and clinically useful surrogate marker for insulin resistance in nondiabetic individuals. PLoS One 11: e0149731, 2016.

22. Relimpio F: 'The relative contributions of insulin resistance and beta-cell dysfunction to the pathophysiology of Type 2 diabetes' by kahn se. Diabetologia 46: 1707, 2003.

23. Tang Q, Li X, Song P and Xu L: Optimal cut-off values for the homeostasis model assessment of insulin resistance (HOMA-IR) and pre-diabetes screening: Developments in research and prospects for the future. Drug Discov Ther 9: $380-385,2015$

24. Morimoto A, Tatsumi Y, Soyano F, Miyamatsu N, Sonoda N, Godai K, Ohno Y, Noda M and Deura K: Increase in homeostasis model assessment of insulin resistance (HOMA-IR) had a strong impact on the development of type 2 diabetes in Japanese individuals with impaired insulin secretion: The Saku study. PLoS One 9: e105827, 2014.

25. Zhu XW, Deng FY and Lei SF: Meta-analysis of atherogenic index of plasma and other lipid parameters in relation to risk of type 2 diabetes mellitus. Prim Care Diabetes 9: 60-67, 2015.
26. Mooradian AD: Dyslipidemia in type 2 diabetes mellitus. Nat Clin Pract Endocrinol Metab 5: 150-159, 2009

27. Tian $\mathrm{L}$ and $\mathrm{Fu} \mathrm{M}$ : The relationship between high density lipoprotein subclass profile and plasma lipids concentrations. Lipids Health Dis 9: 118, 2010

28. Tian L, Liu Y, Qin Y, Long S, Xu Y and Fu M: Association of the low-density lipoprotein cholesterol/high-density lipoprotein cholesterol ratio and concentrations of plasma lipids with high-density lipoprotein subclass distribution in the Chinese population. Lipids Health Dis 9: 69, 2010

29. Wong WT, Wong SL, Tian XY and Huang Y: Endothelial dysfunction: The common consequence in diabetes and hypertension. J Cardiovasc Pharmacol 55: 300-307, 2010.

30. Matsumoto T, Lopes RA, Taguchi K, Kobayashi T and Tostes RC: Linking the beneficial effects of current therapeutic approaches in diabetes to the vascular endothelin system. Life Sci 118: 129-135, 2014.

31. Kalani M: The importance of endothelin-1 for microvascular dysfunction in diabetes. Vasc Health Risk Manag 4: 1061-1068, 2008.

32. Finch J and Conklin DJ: Air pollution-induced vascular dysfunction: Potential role of endothelin-1 (ET-1) system. Cardiovasc Toxicol 16: 260-275, 2016

33. Ergul A: Endothelin-1 and diabetic complications: Focus on the vasculature. Pharmacol Res 63: 477-482, 2011

34. Kurohane Kaneko Y and Ishikawa T: Dual role of nitric oxide in pancreatic $\beta$-cells. J Pharmacol Sci 123: 295-300, 2013.

35. Xie W and Du L: Diabetes is an inflammatory disease: Evidence from traditional chinese medicines. Diabetes Obes Metab 13: 289-301, 2011.

36. Ziamajidi N, Nasiri A, Abbasalipourkabir R and Sadeghi Moheb S: Effects of garlic extract on TNF- $\alpha$ expression and oxidative stress status in the kidneys of rats with STZ + nicotinamide-induced diabetes. Pharm Biol 55: 526-531, 2017.

37. Zhao L, Sun T and Wang L: Chitosan oligosaccharide improves the therapeutic efficacy of sitagliptin for the therapy of chinese elderly patients with type 2 diabetes mellitus. Ther Clin Risk Manag 13: 739-750, 2017

38. Liu Y, Wang X, Zhao Y, Zhao P, Wang L, Zhai Q, Zhang X, Tian W, Xiang $\mathrm{X}$ and $\mathrm{Li} \mathrm{T}$ : Upregulation of tumor necrosis factor- $\alpha$-induced protein 8-like $2 \mathrm{mrna}$ is negatively correlated with serum concentrations of tumor necrosis factor- $\alpha$ and interleukin 6 in type 2 diabetes mellitus. J Diabetes Res 2017: 4802319, 2017.

39. Zhang Y, Proenca R, Maffei M, Barone M, Leopold L and Friedman JM: Positional cloning of the mouse obese gene and its human homologue. Nature 372: 425-432, 1994.

40. Hosoi T and Ozawa K: Possible pharmacological approach targeting endoplasmic reticulum stress to ameliorate leptin resistance in obesity. Front Endocrinol (Lausanne) 7: 59, 2016.

41. Jacobo-Cejudo MG, Valdes-Ramos R, Guadarrama-Lopez AL, Pardo-Morales RV, Martinez-Carrillo BE and Harbige LS: Effect of n-3 polyunsaturated fatty acid supplementation on metabolic and inflammatory biomarkers in type 2 diabetes mellitus patients. Nutrients 9: E573, 2017.

42. Catalan V, Gomez-Ambrosi J, Rodriguez A, Salvador J and Fruhbeck G: Adipokines in the treatment of diabetes mellitus and obesity. Expert Opin Pharmacother 10: 239-254, 2009.

43. Erdem G, Dogru T, Tasci I, Sonmez A and Tapan S: Low plasma apelin levels in newly diagnosed type 2 diabetes mellitus. Exp Clin Endocrinol Diabetes 116: 289-292, 2008.

44. Zhu S, Sun F, Li W, Cao Y, Wang C, Wang Y, Liang D, Zhang R, Zhang S, Wang H and Cao F: Apelin stimulates glucose uptake through the PI3K/Akt pathway and improves insulin resistance in 3T3-L1 adipocytes. Mol Cell Biochem 353: 305-313, 2011.

45. Shen X, Zhou N, Mi L, Hu Z, Wang L, Liu X and Zhang S: Phloretin exerts hypoglycemic effect in streptozotocin-induced diabetic rats and improves insulin resistance in vitro. Drug Des Devel Ther 11: 313-324, 2017

46. Williams LJ, Nye BG and Wende AR: Diabetes-related cardiac dysfunction. Endocrinol Metab (Seoul) 32: 171-179, 2017.

47. Kang OH, Shon MY, Kong R, Seo YS, Zhou T, Kim DY, Kim YS and Kwon DY: Anti-diabetic effect of black ginseng extract by augmentation of AMPK protein activity and upregulation of GLUT2 and GLUT4 expression in db/db mice. BMC Complement Altern Med 17: 341, 2017 